\title{
Polycystic Ovary Syndrome (PCOS) is associated with increased risk of late but not early pregnancy loss after IVF. (Mini-commentary on BJOG-20-1651.R1)
}

\author{
Adam H Balen ${ }^{1}$ \\ ${ }^{1}$ Leeds Teaching Hospitals NHS Trust
}

October 29, 2020

Mini-commentary on BJOG-20-1651.R1: Early and late pregnancy loss in women with polycystic ovary syndrome undergoing IVF/ICSI treatment: a retrospective cohort analysis of 21,820 pregnancies

Polycystic Ovary Syndrome (PCOS) is associated with increased risk of late but not early pregnancy loss after IVF

Adam H Balen,

Leeds Teaching Hospitals, UK

Polycystic Ovary Syndrome (PCOS) is the commonest cause of subfertility, although IVF should be the treatment "of last resort" after lifestyle modification and ovulation induction (Balen, et al Human Reproduction Update 2016; 22: 687-708). There are a number of PCOS phenotypes and associated endocrine and metabolic disturbances, which in turn vary across ethnicities (Teede et al Fertil Steril 2018;110 : 364-379). Whilst PCOS is associated with increased risk of miscarriage and pregnancy complications, the question is whether this is secondary to PCOS itself, associated comorbidities or the mode of conception.

A study of metabolic complications of pregnancy in 14,882 PCOS women compared with 9,081,906 controls found women with PCOS to be more obese $(22.3 \%$ vs. $3.5 \%, \mathrm{P}<0.001)$, with pre-gestational diabetes $(4.1 \%$ vs. $0.9 \%, \mathrm{p}<0.001)$ and chronic hypertension $(8.4 \%$ vs. $1.8 \%, \mathrm{P}<0.001)$. Those with PCOS were more likely to develop gestational diabetes, pregnancy associated hypertension and pre-eclampsia (Mills et al, Human Reproduction 2020; 35 : 1666-1674).

The current study from China (He et al, BJOG xxxx ) assessed pregnancy outcomes in 21,820 women who conceived after IVF, of whom 2,357 (10.8\%) had PCOS. After adjustment for age and other confounders the rate of early pregnancy loss (EPL), prior to 13 weeks, was not associated with PCOS (16.6\% versus $18.3 \%$, OR 0.89, 95\% CI, 0.79-0.99,P =.04). Whereas those with PCOS demonstrated a higher rate of late pregnancy loss (LPL) (6.4\% vs 3.6\%, OR 1.81, 95\% CI, 1.48-2.21,P<.001). In particular overweight and obese PCOS women had higher odds of LPL (OR, 2.18; 95\% CI, 1.48-3.21 and OR, 2.68; 95\% CI, 1.47-4.90) compared with normal weight women.

As might be expected women with PCOS were more likely to be overweight and obese than those without PCOS $(32.4 \%$ vs $16.1 \%$, and $7.4 \%$ vs $2.2 \%, P<.001)$ and had higher rates of hypertensive disease and diabetes mellitus (5.9\% vs $1.2 \%$ and $2.7 \%$ vs $0.2 \%$ ). Regardless of gestational age and PCOS status, the odds of having a pregnancy loss was higher in all women with an elevated BMI, especially in the second trimester.

It is important to consider the distinction between natural and assisted conception, and also the different aetiologies for early and late pregnancy loss, with this study indicating an elevated BMI and comorbidities in 
the PCOS women leading to an increased risk of LPL. A number of studies have also identified an interaction between ethnicity, PCOS phenotype and reproductive outcomes, and so this study of predominantly Chinese women may not be representative of all women with PCOS.

Lifestyle modification and health optimisation prior to pregnancy and fertility treatment is of paramount importance for all women both to reduce any risks during pregnancy and improve the long-term outcome for the baby. This study reinforces the additional risks for women with PCOS, which may be largely but not exclusively attributable to being overweight. The approach should ideally be long-term and sustained, as "quick fix" weight loss programmes are unlikely to substantially alter the ability to achieve a successful pregnancy through IVF (Norman \& MolFertil Steril 2018; 110 : 581-586).

Disclosure of interest: Dr. Balen reports personal fees from Genesis Health Care LLP; Leeds Fertility; personal fees from Balance Reproductive Health Ltd.; non-financial support from WHO GDG; non-financial support from RCOG Council; non-financial support from British Fertility Society; non-financial support from Lecturing - widely; non-financial support from Medical advisor to Verity; non-financial support from CRE-PCOS; non-financial support from FIGO, outside the submitted work.

A completed disclosure of interest form is available to view online as supporting information. 